https://doi.org/10.22319/rmcp.v9i3.4500

Nota de investigación

\title{
Oportunidades para caprinocultores de Guanajuato, México, en la comercialización de queso fino
}

\section{Opportunities for goat farmers in Guanajuato, Mexico, in the marketing} of fine cheese

Rodolfo Santos-Lavalle ${ }^{\mathrm{a}}$

Juan José Flores-Verduzco ${ }^{\mathrm{a}}$

Fernando Cervantes-Escoto ${ }^{\mathrm{a}}$

José María Salas-González

Leticia Myriam Sagarnaga-Villegas ${ }^{c *}$

${ }^{a}$ Centro de Investigaciones Económicas Sociales y Tecnológicas de la Agroindustria y la Agricultura Mundial (Ciestaam). Universidad Autónoma Chapingo (UACh). Km 38.5 Carretera México -Texcoco, Chapingo, Estado de Mexico. México.

${ }^{\mathrm{b}}$ Departamento de Sociología Rural, Chapingo, Estado de Mexico. México.

${ }^{\mathrm{c}}$ Departamento de Zootecnia, Chapingo, Estado de Mexico. México.

*Autor de correspondencia: sagarnaga.myriam@gmail.com

\section{- Resumen:}

A partir de 2008 el precio real de la leche de cabra se ha mantenido a la baja. Ante la imposibilidad de negociar una mejora, algunos caprinocultores han optado por transformarla en queso artesanal. Esta investigación permite conocer los retos y oportunidades al tomar esta decisión. El objetivo fue analizar las causas que limitan la participación y el posicionamiento en el mercado de empresas de caprinocultores productoras de queso fino, ubicadas en Guanajuato, México, con el fin de identificar alternativas orientadas a incrementar ventas. El estudio se realizó bajo el enfoque de red de 
valor y se aplicaron encuestas semi-estructuradas a los diferentes actores que la integran; la información permitió identificar flujos comerciales, formación de precios, problemas percibidos y su complejo causal. Se encontró que las empresas tienen débil posicionamiento en los mercados y limitada participación en venta directa a detallistas en mercados potenciales. La escasa diferenciación de productos y el bajo poder de negociación con sus compradores son las principales causas. A partir de esos hallazgos, se propone gestionar innovaciones en productos, procesos y mercadotecnia.

- Palabras clave: Red de valor, Diferenciación de productos, Posicionamiento, Innovación.

\begin{abstract}
- Abstract:
Since 2008 the real price of goat's milk has been decreasing. By the impossibility of negotiate an improvement, some producers have chosen to make artisan cheese. This research allows to know the challenges and opportunities to take this decision. The objective was to analyze the causes that limit the participation and the positioning in the market of companies producers of fine cheese owned by goat producer, located in Guanjuato, México, in order to identify alternatives aimed at increasing sales. The study was conducted under the value network approach and semi-structured surveys were carried out to different actors that make it up; the information allowed to identify trade flow, price formation and identify perceived problems and their causal complex. It was found that the analized enterprises have a weak position in the markets and a limited participation in direct sales to retailers in potential markets. The low differentiation of the products and the low bargaining power with its buyers are the main causes. Based on these findings, it is proposed to manage innovations in products, processes and marketing.
\end{abstract}

Key words: Value network, Product differentiation, Positioning, Innovation.

Recibido 27/05/2017

Aceptado 23/11/2017

En México el estado de Guanajuato produce $27.3 \%$ de la leche de cabra, ocupando el segundo lugar nacional ${ }^{(1)}$. En esa entidad existen dos grupos de empresas productoras de queso fino: aquéllas propiedad de caprinocultores que transforman su propia leche, y las que al no tener producción primaria, se abastecen de centros de acopio. 
Los mercados de la leche están monopolizados ${ }^{(2)}$, razón por la cual los productores tienen bajo poder de negociación, confirmado con una tendencia del precio real a la baja durante ocho años consecutivos: de $\$ 7.42$ por litro en 2008 , a $\$ 4.95$ en $2016^{(1)}$. Por ello encuentran en la fabricación de quesos una alternativa para mejorar sus ingresos.

La investigación se focaliza en siete empresas que pertenecen al primer grupo, las cuales llevan más de 20 años en la actividad. Sin embargo, no han podido mantener un crecimiento en ventas, por lo que, en la actualidad, $71 \%$ de ellas reportan infraestructura subutilizada. Mientras esto pasa, empresas competidoras, ubicadas en la misma entidad y en el estado de Querétaro, están ampliando instalaciones y conquistando nuevos clientes. Por tal motivo el objetivo de este estudio fue analizar las causas que limitan la participación y el posicionamiento en el mercado de estas empresas (propiedad de productores), con el fin de identificar alternativas orientadas a incrementar el volumen de ventas.

Se tomó como guía metodológica el concepto de red de valor, propuesto por Nalebuf y Branderburguer ${ }^{(3)}$ y adaptado para el sector agropecuario por Muñoz ${ }^{(4)}$. Este enfoque permite a una, o a un grupo de empresas de un sector, tomar acciones específicas en el marco de su entorno competitivo, pues ayuda a comprender las acciones de los demás actores y las reglas del juego que emplean, tomando en cuenta las fuerzas competitivas que deben enfrentar ${ }^{(5)}$, y así diseñar o reestructurar su modelo de negocios.

La red de valor está concebida como "el conjunto de empresas especializadas en una actividad en común, caracterizada por la concentración territorial de sus actores, con desarrollo de vínculos de naturaleza económica o no, que contribuyen de esta manera a la generación de valor o riqueza, tanto para sus miembros como su territorio"(3). Esta se conforma por cinco grupos de actores que es necesario analizar por separado y en sus interacciones; en el centro se ubican empresas cuyo poder les permite configurar la dinámica de la red de valor, conocidas como empresas tractoras ${ }^{(4)}$.

La investigación se efectuó de mayo de 2016 a enero de 2017. El trabajo de campo se realizó con apoyo de un facilitador, quien referenció nombre y ubicación de las empresas. Se aplicaron entrevistas semi-estructuradas a los propietarios de las siete empresas bajo estudio, es decir se realizó un censo. La selección del resto de actores se hizo por muestreo no probabilístico, conocido como bola de nieve $\mathrm{e}^{(6)}$, para ello se solicitó a los primeros que mencionaran otros actores con los que se relacionan.

Se entrevistaron doce proveedores de leche de cabra independientes; cuatro dueños o gerentes de empresas productoras de queso, consideradas como competidores. También un funcionario del sistema producto caprino estatal, otro de la Secretaria de Desarrollo Agropecuario de Guanajuato, cuatro investigadores y asesores en cabras; todos estos considerados como complementadores. Un actor cumple con esta función, si las acciones desarrolladas por él permiten que los clientes valoren más los bienes y servicios ofrecidos, 
o bien que los proveedores prefieran relaciones con la empresa tractora en vez de dirigirse con la competencia ${ }^{(4)}$. Adicionalmente se entrevistó a dos distribuidores de queso de cabra $\mathrm{y}$ at personal de tres distintos puntos de venta al detalle en la Ciudad de México, considerados dentro del grupo de clientes.

La información cuantitativa recabada se analizó utilizando estadística descriptiva y la cualitativa resultó útil en la construcción de flujos e identificación de actores, el análisis de la red de valor y la elaboración del árbol de problemas.

Para construir el árbol de problemas y su complejo causal se aplicó la metodología del marco lógico ${ }^{(7)}$. Finalmente, se generó un conjunto de propuestas para el problema principal, con acciones que eliminan las causas que lo originan.

La leche producida en granja tiene el siguiente flujo: pasa por un proceso de acopio y comercialización cuando las empresas requieren comprarla, de lo contrario, al ser producida en granja propia pasa directamente a la quesería para su transformación.

Las empresas incluidas en este estudio procesan 103,904 L mensuales de leche y obtienen $16,468 \mathrm{~kg}$ de queso. Este producto se distribuye por tres canales de comercialización: venta directa a detallistas en centros de consumo, venta a distribuidores y venta a consumidores locales (Figura 1).

Figura 1: Flujo comercial del queso de cabra en las empresas de caprinocultores

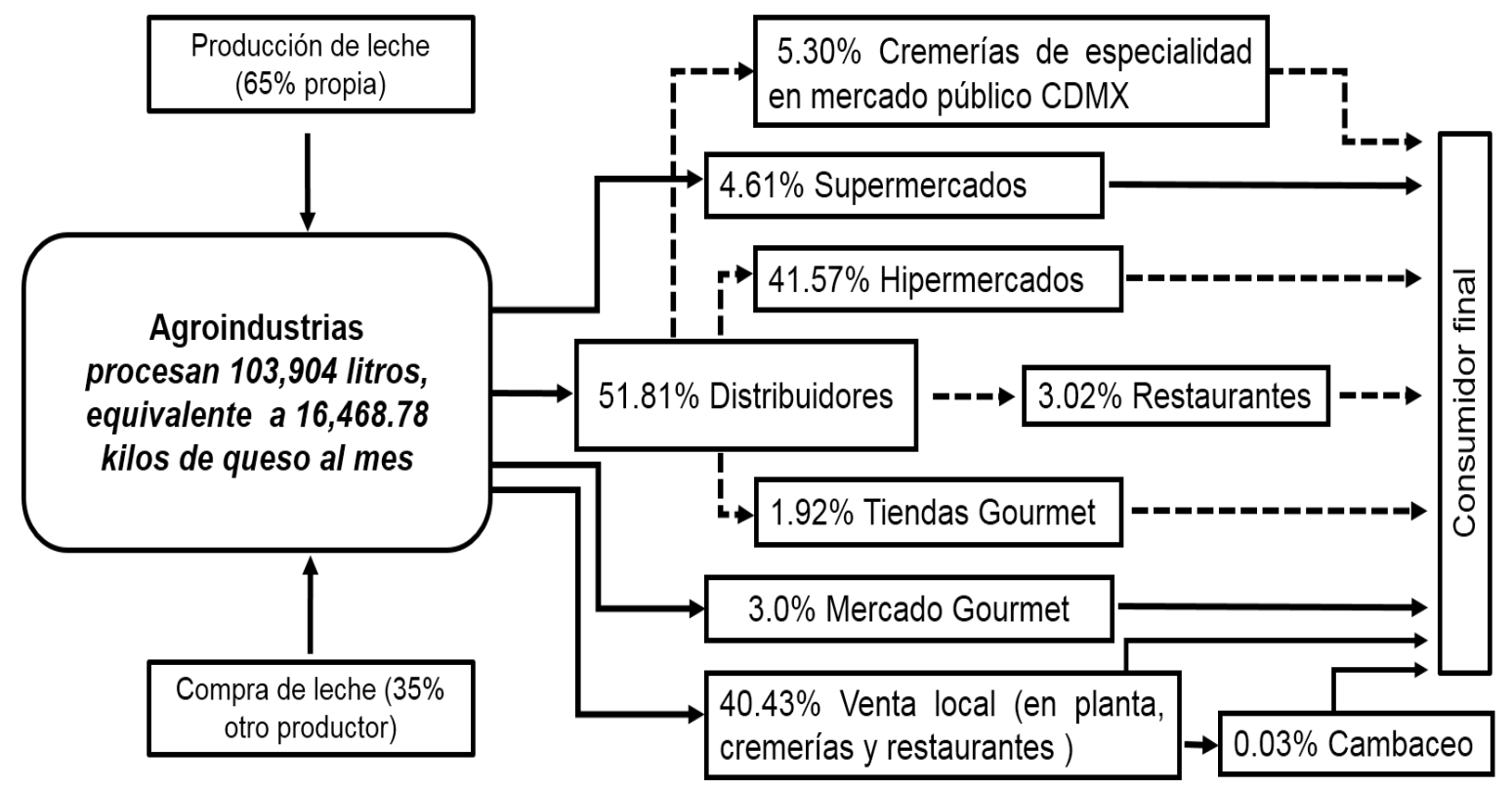

Fuente: elaboración propia con datos de campo, 2016. 
En el flujo comercial se identificaron los siguientes actores: productores de leche, agroindustrias queseras, distribuidores, cremerías, supermercados, hipermercados, restaurantes, mercados y tiendas gourmet, detallista de "cambaceo" y consumidor final.

De acuerdo al volumen comercializado, las empresas dependen en gran medida de los distribuidores $(51.81 \%)$ para que el producto llegue a los detallistas; sin embargo, también se observa una estrategia para trabajar en circuitos cortos de comercialización, vendiendo directamente a detallistas e incluso al consumidor final (48.19\%), con ello se busca acceder a una mayor proporción del valor generado ${ }^{(8)}$. Los precios pagados por el consumidor final no solo se ven influenciados por el grado de intermediación, también dependen de la máxima cantidad de dinero que están dispuestos a desembolsar. Por ejemplo, el mismo producto, puesto en un mercado gourmet recibe un precio mayor (\$323.00) pues ahí concurren consumidores con alto poder adquisitivo (Cuadro 1). Un caso especial es la venta por "cambaceo", realizada por detallistas que entregan el producto a domicilio a $\$ 375.00$ $\mathrm{kg}^{-1}$.

Cuadro 1: Precios pagados en función del tipo de mercado en que participan las empresas

\begin{tabular}{lccc}
\hline \multirow{2}{*}{ Tipo de mercado detallista } & \multicolumn{3}{c}{ Precio por kilogramo de queso (pesos) } \\
\cline { 2 - 4 } & Distribuidor & Detallista & Consumidor final \\
\hline Cremerías CDMX & 130.00 & 167.00 & 225.00 \\
Supermercados & $\mathrm{N} / \mathrm{A}$ & 148.50 & 245.00 \\
Venta local & $\mathrm{N} / \mathrm{A}$ & $\mathrm{N} / \mathrm{A}$ & 165.00 \\
Hipermercados & 115.00 & 175.00 & 265.00 \\
Tiendas gourmet & 140.00 & 182.00 & 265.00 \\
Mercado gourmet & $\mathrm{N} / \mathrm{A}$ & 140.00 & 323.00 \\
Casa por casa & $\mathrm{N} / \mathrm{A}$ & 165.00 & 375.00 \\
\hline
\end{tabular}

$\mathrm{N} / \mathrm{A}=$ no aplica.

También se encontró que el consumidor final se beneficia del menor precio cuando las empresas realizan la venta local ( $\$ 165.00$ por $\mathrm{kg}$ ), evidencia de los circuitos cortos de comercialización, que reduce costos y a la vez los precios finales de los alimentos ${ }^{(9)}$.

Por su parte el margen del precio final que es retenido por los distintos actores en el flujo comercial depende tanto de lo que paga el consumidor final, como de los niveles de intermediación (Cuadro 2). Con relación a los detallistas, se observa variación, pero los que venden casa por casa y en mercados gourmet retienen incluso más dinero que las propias empresas productoras de queso. En cambio, los distribuidores se quedan con la menor cantidad, compensada por el volumen desplazado, sobre todo en hipermercados. Las empresas logran un margen mayor cuando realizan venta local. 
Cuadro 2: Margen absoluto retenido por los actores en el flujo comercial (pesos/kilogramo)

\begin{tabular}{lcccc}
\hline Tipo de mercado detallista & $\begin{array}{c}\text { Precio al } \\
\text { consumidor final }\end{array}$ & $\begin{array}{c}\text { Consumidor- } \\
\text { detallista }\end{array}$ & $\begin{array}{c}\text { Detallista- } \\
\text { distribuidor }\end{array}$ & $\begin{array}{c}\text { Detallista o } \\
\text { distribuidor-empresa }\end{array}$ \\
\hline Cremerías CDMX & 225.00 & 58.00 & 37.00 & 130.00 \\
Supermercados & 245.00 & 80.00 & $\mathrm{~N} / \mathrm{A}$ & 148.50 * \\
Venta local & 165.00 & $\mathrm{~N} / \mathrm{A}$ & $\mathrm{N} / \mathrm{A}$ & 165.00 \\
hipermercado & 265.00 & 90.00 & 60 & 115.00 \\
Tiendas gourmet & 265.00 & 83.00 & 42.00 & 140.00 \\
Mercado gourmet & 323.00 & 183.00 & $\mathrm{~N} / \mathrm{A}$ & 140.00 \\
Casa por casa & 375.00 & 210.00 & $\mathrm{~N} / \mathrm{A}$ & 165.00 \\
\hline
\end{tabular}

${ }^{*}$ La diferencia son descuentos $(10 \%)$ realizados por la tienda de autoservicio.

La descripción y el análisis de la red de valor inician en las empresas tractoras (Figura 2). Estas tienen en promedio 22 años elaborando queso de cabra, con un rango de 16 a 40 años. La escala va de 2,000 a 72,000 L al mes, algunas ocupan mano de obra contratada adicional a la familiar, utilizan $100 \%$ de leche de cabra evitando el uso de extensores. Comercializan los productos con diferentes marcas. Se puede afirmar que fabrican queso de manera artesanal, la producción es manual y se respeta el conocimiento sobre el "saber hacer" que se ha importado directamente de Francia.

Figura 2: Representación de la red de valor con sus interrelaciones

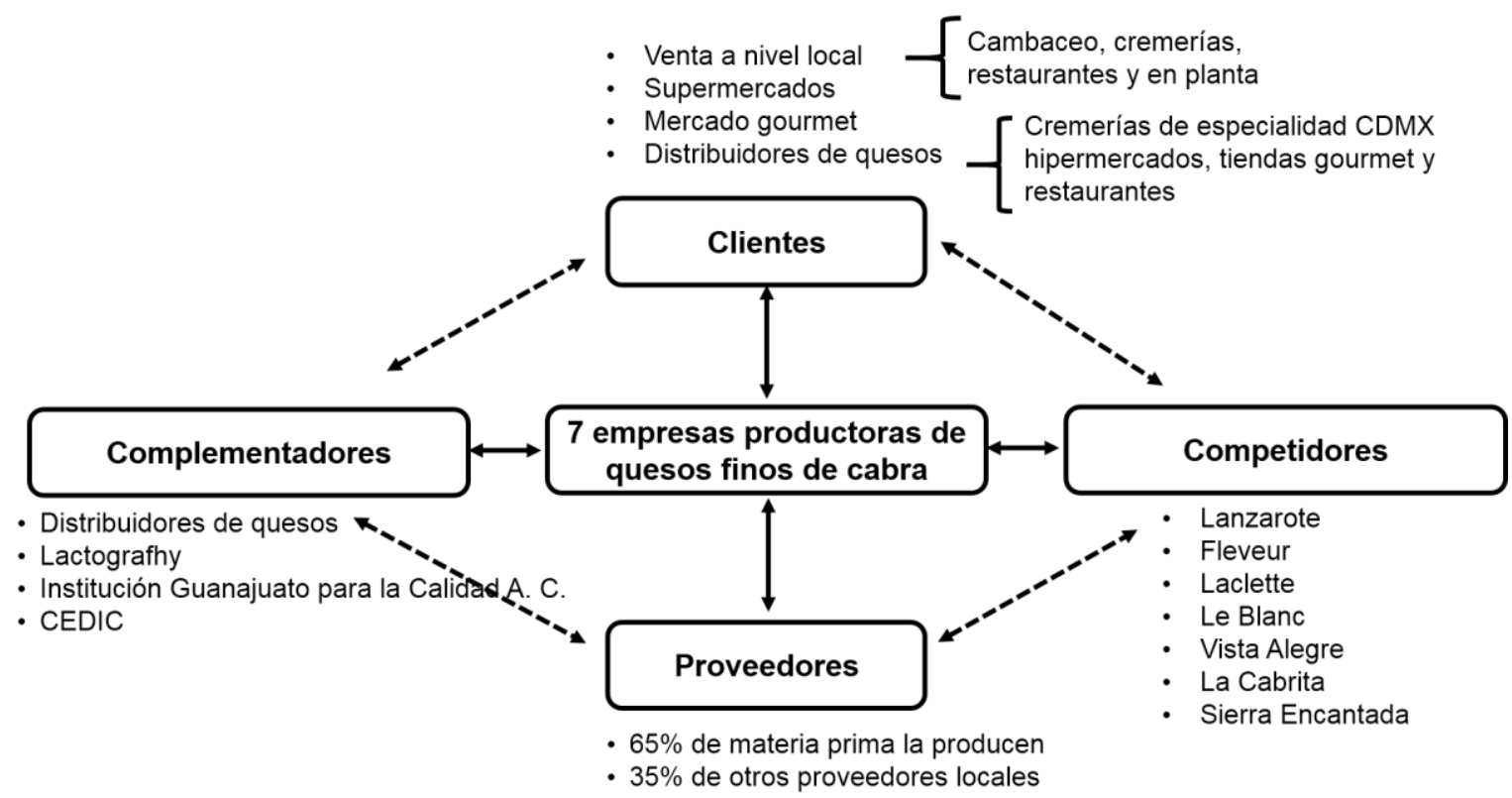

Fuente: elaboración propia con datos de campo, 2016. 
Tres empresas se encuentran en expansión, tres más se consideran estancadas y una en retroceso debido a que ha reducido su capacidad de operación. Dos de las que se encuentran en expansión operan con mano de obra contratada y orientan la oferta hacia mercados que demandan mayor volumen; otra dirige su oferta al mercado gourmet. Se ha documentado en quesos artesanales de leche de vaca que cuando las empresas evolucionan en escala y perciben un panorama favorable en la demanda, es cuando utilizan estrategias de comercialización distintas a la venta local ${ }^{(10)}$.

La venta directa a detallistas en centros de consumo tiene como compradores a supermercados y mercados gourmet. Con los supermercados se firma un contrato que establece precio, volumen, frecuencia de abasto y penalizaciones. No hay exclusividad, se pueden encontrar varias empresas compitiendo en una misma tienda. Demandan constancia, estandarización de productos, garantía en calidad y cumplimiento de lo pactado; por ello sólo una empresa vende en este canal, el resto prefiere recurrir a distribuidores y detallistas menos exigentes.

El mercado gourmet es un punto de venta en la Ciudad de México que concentra varios negocios que ofrecen productos dentro de esta categoría. Este es un mercado exigente que selecciona los mejores quesos con base en catación y análisis de los procesos con que se fabrican. Ello explica el alto precio que está dispuesto a pagar el consumidor final. Se venden tres marcas de queso, una de las empresas incluidas en el análisis.

En la venta a distribuidores participan cuatro empresas, no hay contrato establecido, el precio se negocia cuando acuden a la planta o antes del envío. A su vez estos entregan a detallistas: hipermercados, cremerías, tiendas gourmet y restaurantes. Todas las empresas venden localmente en planta y el pago es de contado; además, una de ellas vende en mercados públicos para llegar al consumidor final; otra vende a detallistas que ofrecen productos gourmet; dos venden en cremerías y dos más a restaurantes, todas dentro del estado de Guanajuato.

La materia prima principalmente es producida en granjas propias, ahí se cuida la calidad higiénica mediante buenas prácticas de ordeño, lo cual le confiere garantía al producto. Los productores independientes son seleccionados como proveedores cuando reúnen condiciones de entrega, volumen y calidad demandados. Se ven beneficiados al recibir un mejor precio (\$6.20), comparado con el que recibirían en centros de acopio que abastecen a industrias competidora $(\$ 5.50)$.

En relación con la proveeduría de leche, las empresas tienen disponibilidad todo el año, gracias al control en la reproducción de las cabras. Aun así, existen picos de producción en mayo-junio, que algunas almacenan como pasta láctica o queso semi-maduro. 
Como complementadores, por el lado de la demanda, se ubicó la empresa Lactografhy, quien apoya a productores de queso promocionándolos en sus puntos de venta. También el Instituto Guanajuato para la Calidad A.C. facilita el que algunas empresas implementen Buenas Prácticas de Manufactura (BPM). Un actor puede ejercer dos o más funciones simultáneamente en una red de valor ${ }^{(4)}$, por lo que los distribuidores también se desempeñan como complementadores, ayudando al desarrollo de la demanda. Finalmente, el Centro para el Desarrollo Integral del Campo (CEDIC) ejerce el papel de asesor en el desarrollo de capacidades empresariales con dos empresas.

Los competidores se pueden diferenciar en dos grupos: los de mercado masivo, y los que están en el mercado gourmet. En el primer caso, existe un duopolio que tiene poder para competir por el abasto de leche, fijar reglas de precios y calidad a sus proveedores y dominar en tiendas de autoservicio. Basan su estrategia en la operación de economías de escala (compiten por precio), a diferencia de los quesos artesanales que suelen ser más $\operatorname{costosos}^{(11)}$. También ofrecen otros productos lácteos como yoghurt, jocoque, queso de vaca y de oveja; dan cumplimiento al sistema de Análisis de Riesgos y Control de Puntos Críticos (HACCP) y a las normas de la Food and Drug Administration (FDA); cuentan con innovación de producto; atención y prospección de clientes a través de su fuerza de venta. Su éxito puede confirmarse por la capacidad alcanzada para crecer con nuevas instalaciones o para adquirir a otras empresas competidoras y para exportar pasta láctica de cabra.

En el segundo caso, las empresas tractoras compiten tanto con empresas del duopolio, como con otras dos de menor escala, que se distinguen por producir su propia materia prima y elaborar productos artesanales con leche $100 \%$ de cabra. Una de ellas cuenta con certificación en buenas prácticas de producción, la otra en HACCP y certificación de productos orgánicos. Dirigen su oferta a tiendas especializadas en productos orgánicos y artesanales; ofrecen además leche pasteurizada, yoghurt y cajeta.

El débil posicionamiento de los productos en los mercados se percibe como uno de los problemas a superar por las empresas bajo estudio. Con algunos clientes detallistas lo están perdiendo y con otros no lo han logrado. En el primer caso, una se posicionó desde hace 10 años en cremerías de especialidad de la Ciudad de México; sin embargo, en fechas recientes está perdiendo esa ventaja ante un competidor que ofrece productos diferenciados por un mejor empaque, mayor vida de anaquel y menor tiempo de entrega. En el segundo caso, empresas competidoras en mercados y tiendas gourmet se han posicionado porque comunican e informan al consumidor de mejor manera, resaltando atributos del producto a través de la etiqueta; se diferencian por la certificación orgánica y ofrecen una mayor diversidad de productos.

La falta de diferenciación de las características extrínsecas en los productos se considera la causa principal del débil posicionamiento en los mercados. Factores como el tipo de empaque y la información en la etiqueta (tecnología de proceso, nutricional y el precio) 
desempeñan un papel importante en la elección de compra ${ }^{(12-15)}$. En las empresas analizadas, que participan en cremerías de especialidad, tiendas y mercado gourmet, la información que las etiquetas proporcionan es insuficiente, no mencionan que es un producto gourmet elaborado de manera artesanal, limitando su posicionamiento, razón por la cual están dejando de ganar dinero, pues la disposición a pagar en mercado gourmet puede representar un incremento en precio de $37 \%$, en cremerías de especialidad $25 \%$ y en supermercados $7.5 \%$.

En los supermercados e hipermercados se exige a los proveedores que incluyan código de barras para facilitar el manejo de inventarios dentro de la tienda. Se encontró que cinco de las empresas $(71 \%)$ no lo tienen. Lo anterior imposibilita vender a estos detallistas, que tienen la ventaja de distribuir mayor volumen del producto.

Para estos clientes la calidad también se ha convertido en una condición de compra, y es una forma de discriminar a quienes no cuentan con las certificaciones que la acrediten. Sin embargo, sólo tres empresas (43\%) intentan implementar HACCP, sin contar aún con dicha certificación.

Las empresas bajo estudio no han identificado plenamente el problema de su débil posicionamiento. Sólo perciben que la demanda no ha crecido en los últimos años y que los costos fijos son altos; adicionalmente se sienten presionadas para mantener precios bajos, con el fin de conservar sus clientes.

Otro de los problemas a superar es la limitada participación con venta directa en supermercados e hipermercados; considerados como mercados potenciales, por el volumen que pueden desplazar. Por esta razón resulta estratégico, pues la intervención de distribuidores reduce sensiblemente su participación económica; sin embargo, sólo $4.61 \%$ de la producción tiene este destino.

Para lograr lo anterior las empresa requieren superar las siguientes barreras: el poder para fijar las condiciones de compra a sus proveedores, que les posibilita establecer un precio tope ( $\$ 35.00$ la pieza de $200 \mathrm{~g}$ ); también los requisitos para la aceptación de los productos, tales como uso de código de barras, garantía de certificación de calidad, fechas de abasto con día y hora de entrega; asimismo, tienen autoridad para establecer descuentos a proveedoras por promoción y costo de distribución. Esta situación coincide con lo teorizado por Porter ${ }^{(5)}$, al hablar de que las empresas necesitan contrarrestar las fuerzas competitivas de sus proveedores para mejorar sus utilidades.

Por otro lado, se encontró que cinco de las siete empresas (71 \%) no cuentan con fuerza de ventas en su estructura administrativa, lo que limita su habilidad para negociar y hacer prospecciones que permitan captar más clientes. 
Como productores primarios, las empresas forman parte de la organización "Caprinocultores Unidos de Guanajuato", A. C.; como fabricantes de queso no están organizados, situación que no ayuda a contrarrestar el poder ejercido por los clientes.

Los problemas percibidos y el complejo causal permiten construir el árbol de problemas (Figura 3), en correspondencia con la herramienta de marco lógico ${ }^{(7)}$.

Figura 3: Diagrama de árbol de problemas en las empresas de caprinocultores
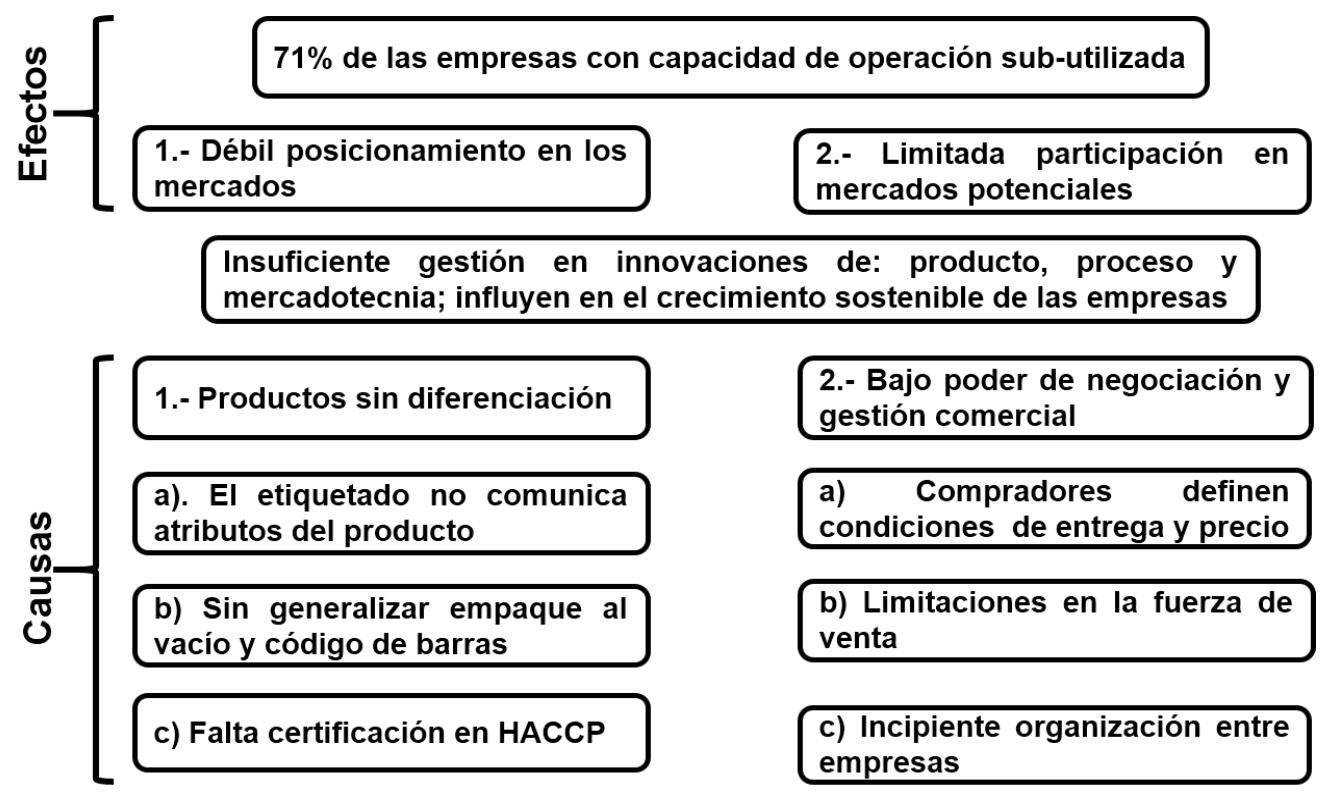

Fuente: elaboración propia con datos de campo, 2016.

Con base en el análisis de esa información, el problema principal es que las empresas no adoptan una estrategia de innovación en producto, procesos y mercadotecnia. Lo anterior fue evidenciado en apartados anteriores al exponer las causas de los problemas percibidos: deficiencias en certificación, código de barras, empaque, etiquetado, capacidad de negociación, fuerza de ventas y organización.

Ese problema tiene como efecto principal que cinco de las siete empresas $(71 \%)$ tienen capacidad instalada subutilizada. En casos extremos sólo se aprovecha $20 \%$ de la misma. Cuando la disponibilidad de materia prima no es en una limitante, la subutilización se atribuye al bajo volumen de ventas.

Para recuperar el posicionamiento en los mercados se sugiere establecer una diferenciación de productos. De conformidad con lo anterior, se propone certificar los procesos de elaboración de queso en HACCP, generalizar el empaque al alto vacío, incluir el código de barras en las etiquetas, rediseñar las etiquetas con el propósito de comunicar a los 
consumidores que el queso es un producto gourmet, elaborado $100 \%$ con leche de cabra de manera artesanal y, finalmente, que es fabricado por productores de leche de cabra, como un mensaje de autenticidad. Comunicar asertivamente información valorada por los consumidores a través de las etiquetas, permite generar confianza, asociación con el lugar y el método de fabricación ${ }^{(16)}$.

También se recomienda establecer un vínculo directo con los consumidores, que se sustente en relaciones de confianza y proximidad ${ }^{(17)}$. Si la búsqueda de esa clientela es de forma individual, la alternativa es colocar a un vendedor profesional en su estructura organizacional. Como algunas empresas no tienen capacidad para contratar fuerza de ventas de manera individual, se propone que se agrupen para generar una alianza estratégica y explorar conjuntamente la contratación de una persona que lleve a cabo esta tarea. Se ha documentado que agruparse con otros productores permite contar con medios suficientes para negociar con las grandes cadenas de distribución y cumplir con sus exigencias ${ }^{(18)}$.

Se concluye que los retos que enfrentan las empresas productoras de quesos finos de cabra son distintos, en función del canal de comercialización en el que se insertan. Cuando orientan la oferta de producto a supermercados e hipermercados, estos presentan barreras de entrada, como el poder de negociación sobre sus proveedores; cuando participan en mercados gourmet, se encuentran con empresas competidoras mejor posicionadas.

Una de las opciones de mercado que ofrece mayores beneficios, tanto para el consumidor de quesos finos de cabra, como para quien los produce, es la venta local, con un menor número de transacciones; sin embargo, no toda la producción puede colocarse por esta vía, por lo que deben buscarse otras alternativas fuera del estado de Guanajuato. Ante esta situación, las empresas productoras de quesos finos requieren definir con claridad a qué mercado dirigirán su oferta, porque lo anterior determina los retos a enfrentar y define las acciones que deberán implementarse.

Si optan por los mercados gourmet, la alternativa es la diferenciación de productos, destacando, además de los atributos sensoriales, el origen y el método de elaboración. En cambio, si la alternativa son los hipermercados y supermercados, las empresas requieren integrarse de manera horizontal, para generar economías de escala y contrarrestar relaciones de poder con sus compradores. 


\section{Agradecimientos}

Se agradece a las empresas que elaboran quesos finos de cabra en Guanajuato, en especial al MVZ Salvador Arellano por el apoyo brindado.

\section{- Literatura citada:}

1. Servicio de Información Agroalimentaria y Pesquera (SIAP). Leche de caprino. Avance mensual de la producción pecuaria. 2016. http://infosiap.siap.gob.mx/repo Avance_siap_gb/pecAvanceProd.jsp. Consultado 16 Dic, 2016.

2. Oseguera MD, Keilbach BMN, Van Der ZA, Sato C, Udo H. "It is better to herd than be herded": making a living with goats in the Bajío region, Mexico. Pastoralism 2014;4(9):1-18.

3. Nalebuff BJ, Branderburger MA. Coo-petencia. Tercera ed. Bogotá, Colombia: Harvard Business School; 1996.

4. Muñoz M, Santoyo H. La red de valor: herramienta de análisis para la toma de decisiones de políticas públicas y estrategia agroempresarial. Chapingo, Estado de México: Universidad Autónoma Chapingo; 2011.

5. Porter EM. Las cinco fuerzas competitivas que moldean la estrategia. En: Ser competitivo. Primera ed. Boston, USA: Harvard Business Review; 2009:3-68.

6. Malhotra NK. Investigación de mercados. Quinta ed. México: Pearson Educación; 2008.

7. Ortegón E, Pacheco JF, Prieto A. Metodología del marco lógico para la planificación, el seguimiento y la evaluación de proyectos y programas. Santiago, Chile: CEPAL; 2005.

8. Craviotti C, Soleno WR. Circuitos cortos de comercialización agroalimentaria: un acercamiento desde la agricultura familiar diversificada en Argentina. Mundo Agrar 2015;16(33):1-19.

9. López GD. Canales cortos de comercialización, un elemento dinamizador. Soberanía Aliment Biodivers y Cult 2012;8:21-24. 
10. Grass-Ramírez JF, Cesín-Vargas A. Situación actual y retrospectiva de los quesos genuinos de Chiautla de Tapia, México. Agric Soc Desarrollo 2014;11(2):201-221.

11. Bouma A, Durham CA, Meunier-Goddik L. Start-up and operating costs for artisan cheese companies. J Dairy Sci 2014;97(6):3964-3972.

12. Park YW. Goat milk products: quality, composition, processing, and marketing. In: Duane E. et al, editors. Encyclopedia of animal science. Second ed. USA: CRC Press; 2011:541-545.

13. Lancu RM. Consumers perception and behavior towards ecosanogene products made by goat milk. Manag Sustain Dev Subiu, Rum 2014;6(2):87-92.

14. Yee Lee P, Lusk K, Mirosa M, Oey I. Effect of information on Chinese consumers perceptions and purchase intention for beverages processed by high pressure processing, pulsed-electric field heat treatment. Food Qual Prefer 2014;40:16-23.

15. Musto M, Cardinale D, Lucia P, Faraone D. Influence of different information presesentation formats on consumer acceptability: the case of goat milk presented as obtained from different rearing systems. J Sens Stud 2015;30:85-98.

16. Renting H, Marsden T, Banks J. Understanding alternative food networks : exploring the role of short food supply chains in rural development. Environ Plan 2003;35:393411.

17. González CI, De Haro GT, Ramos RE, Renting H. Circuitos cortos de comercialización en Andalucía: un análisis exploratorio. Rev Española Estud Agrosociales Pesq 2012;(232):193-227.

18. Sales I, Campos A. Un sistema de distribución un sistema de producto. Soberanía Aliment Biodivers Cult 2012;8:7-9. 